Chapter 3

\title{
The Tricuspid valve
}

Francesco F Faletra, Laura A Leo, Vera L. Paiocchi, Stefanos Demertzis, Giovanni Pedrazzini, and Siew Yen Ho

\section{Introduction}

Interest on anatomy of tricuspid valve (TV) has increased in the last two decades with the awareness that functional tricuspid regurgitation (FTR) is not an innocuous bystander of left-side heart disease but, on the contrary, an insidious disease progressively leading to untreatable right heart failure and eventually to death. The majority of cases of severe tricuspid regurgitation are functional due to right ventricular (RV) enlargement, annular dilation, and leaflet tethering. Commonly, RV dilatation is secondary to left-side valvular diseases, (mainly mitral valve stenosis or regurgitation), heart failure and RV volume or pressure overload. Less frequently, severe FTR is the consequence of tricuspid annular dilatation due to isolated atrial enlargement caused by atrial fibrillation. Mild tricuspid regurgitation in the setting of structurally normal tricuspid valve is a normal echocardiographic aspect.

A thorough knowledge of the anatomical architecture of the TV and its spatial relationships with the surrounding structures may help to appreciate the process through which FTR occurs. In line with the other chapters, this chapter describes the normal anatomy of TV as it is revealed by computed tomography (CT), cardiac magnetic resonance (CMR) and two dimensional and tridimensional transthoracic (2D-3D TTE) and transesophageal echocardiography (2D-3D TEE). We finish this chapter with a brief description of pathoanatomy of FTR. 


\section{Anatomy of tricuspid valve}

\section{General concepts}

The TV, positioned at the right atrio-ventricular junction, is the largest and the most apically-positioned valve, oriented at approximately $45^{\circ}$ to the sagittal plane. Although exposed to much lower systolic pressure, the global architecture of TV is surprisingly similar to MV. The complex valve apparatus consists, in fact, of three leaflets, a circumferential hinge line where leaflets are suspended and a suspension system composed of chordae tendineae attached to papillary muscles or to the septum. However, contrary to MV where the architectural configuration is almost similar among different individuals, the TV presents numerous anatomical variations in number of leaflets and papillary muscle arrangement. Furthermore, there is much discussion on naming of the leaflets specifically on whether a more attitudinal nomenclature should be adopted. While the septal leaflet is precisely just that, the anterior leaflet is located more antero-superiorly while the posterior leaflet is more posterior-inferior. In keeping with common usage, enabling team members to understand each other, we maintain the classical nomenclature of septal, anterior and posterior for naming the leaflets. Also in keeping with the chapter of MV, we describe each component of the TV apparatus separately.

\section{Tricuspid Annulus}

The tricuspid annulus (TA) conceptualized as a discrete ring of dense connective from which tricuspid leaflets are suspended, simply does not exist. Indeed, the right atrio-ventricular junction, is made up of a juxtaposition of atrial and ventricular myocardium, separated by epicardial fibro-adipose tissue (EAT) of the atrio-ventricular groove and the hinge line of the tricuspid leaflets. However, for clarity and consistency with the literature, we continue to name this part of the right atrio-ventricular junction as "annulus".

It is worth emphasizing that EAT is an integral part of the TA. This tissue of the atrio-ventricular groove comprising of adipocytes intermingled in a network of thin collagen and elastic fibers, extends up to

the hinge line of tricuspid leaflets. With the exclusion of the small septal area, where the hinge line of septal leaflet intersects the central fibrous body and divides the membranous septum into two parts (see below), the EAT covers the entire anterior-lateral-posterior C-shaped groove and directly joins the leaflets. The connective fibers of the leaflet fibrosa tapers off into the fibro-elastic network of the EAT, the atrial and ventricular walls (Fig. 3.1 A, B). This particular anatomical arrangement, assures an 
excellent electrical insulation between the atrial and the ventricular myocardium, leaving the atrioventricular conduction system, the only muscular tissue crossing this barrier of insulation.

Echocardiography may potentially identify the EAT which, when extremely abundant, shows a characteristic hourglass appearance. However, though the atrio-ventricular groove contains relatively abundant EAT, the nearly similar acoustic impedance makes rather difficult differentiation between EAT and the surrounding muscular tissue (Fig 3.1 C, D). CT can also identify the EAT from atrium and ventricular walls, because EAT has a lower density than the muscular tissue on $\mathrm{x}$-ray and it is represented on CT imaging as darker area. In reality, despite the highest spatial resolution (voxel $=0.6$ $\mathrm{mm}$ ), borders of the dark area corresponding to EAT and borders of surrounding muscular structures remain rather indistinct (Fig. 3.1 E, F).

CMR balanced steady-state free procession (SSFP) sequences, is ideal for a perfect delineation of the EAT in the atrio-ventricular groove and its extension up to the hinge line. In this sequence, in fact, blood and the adipose tissue produce a very high signal. Conversely, the signal originating from the muscular tissue is weak. The sequence therefore allows clear visualization of the EAT located in the atrioventricular groove (Fig. 3.2).

\section{Surgical aspect}

The hinge between leaflets and right atrium (RA) is clearly delineated by different colors: the wall of the right atrium is rather pale pink while the leaflets have a yellowish white color. Sutures are usually placed on the atrial wall $2 \mathrm{~mm}$ from the hinge line (to avoid damage to leaflets) and directed towards the ventricle to avoid the coronary artery.

From a surgical point of view, TA can be described in terms of four segments: the aortic, anterior, posterior and septal segments. This segmentation can be best understood by using 3D TEE technique which allows the entire contour of TA to be visualized in "en face" perspective. The figure 3.3, shows side-by-side the anatomic specimens (A, B) and the corresponding 3D TEE imageswith the surgical segmentation of the TA (dotted circles) superimposed.

The aortic segment, adjacent to the aortic root, is the hinge line where the anterior part of the septal leaflet and the anterior leaflet are suspended and includes the antero-septal commissure. Identification of this segment in the operating room is of paramount relevance. Indeed, only a few millimeters (occupied by the transverse pericardial sinus) separate this segment from the aortic root (Fig. 3.3 B, D). 
During surgical repair or replacement with a prosthesis, imprecise suturing may damage the aortic leaflet or the right coronary aortic sinus. Moreover, due to the strict anatomical proximity, large aneurysms or dilatation of the aortic root may alter the geometry of the TA, causing tricuspid regurgitation. Similarly, endocarditis or abscesses of the posterior area of the aortic root may invade the TV.

Anterior and posterior segments are related to the free wall of the right ventricle. As described above, because of the absence of any rigid support, the TA has the tendency of lengthening and enlarging along these segments. Conversely, the septal segment being firmly attached to the membranous septum and reinforced by right fibrous trigone in the vicinity of antero-septal commissure is relatively spared from annular dilatation. The atrioventricular conduction system is located within the septal segment. In consideration of this fact, the surgical rings are designed as "incomplete" rings, missing the septal segment, to avoid injuring the conduction system when placing sutures to secure the ring (Fig 3.4).

\section{Shape and motion of TA}

TA is almost oval (though in diastole it tends to be grossly circular). Similar to the mitral annulus but less accentuated, the shape of TA is not planar but roughly resembles a "saddle" configuration with two superiorly displaced regions corresponding to the anterior-septal and posterior-lateral segments and two regions displaced inferiorly corresponding to the anterior-lateral and posterior-septal segments (near the ostium of coronary sinus). This non-planar configuration is a consequence of several factors: the bulging of the right outflow tract, the undulating course of anterior and posterior segments and the position of septal segment which is attached lower towards the apex. Indeed, anatomical specimens clearly demonstrate the non-linear hinge line of the septal leaflet. It is undulating being most apical at a point close to the mid-portion of the leaflet's hinge and ascends toward the antero-septal segment, peaking at the antero-septal commissure (Fig.3.4 C,D). The complex non-planarity of TA requires a 3D imaging modality for assessing its real size. Accordingly, in healthy individuals the most recent data on TA size are derived from 3D echocardiography (the mean maximal circumference and area are $12 \pm 1$ $\mathrm{cm}$ and $11 \pm 2 \mathrm{~cm}^{2}$ ) or from CT (maximum diastolic area of $10.7 \mathrm{~mm}^{2} \pm 2.2 \mathrm{~cm}^{2}$ ). Notably, the 3D measurements are smaller than those made on $\mathrm{CT}$, likely due to differences in spatial resolution, which is higher with CT than with 3D (isotropic voxel $=0.6 \mathrm{~mm}$ versus anisotropic voxel $>1 \mathrm{~mm}$ respectively). 
Interestingly, although in literature these measurements referred to the TA, in reality they were taken on the hinge line of tricuspid leaflets.

TA has two fundamental motions: a sphincteric contraction and an excursion towards the apex.

The sphincteric motion occurs because the anterior and posterior leaflets are attached directly on right ventricular myocardium following its systolic contraction. TA size (area, perimeter, and dimensions) progressively decrease during systole reaching a minimum size in late systole and a maximum size in late diastole after atrial contraction. The contraction of the TA results in $20 \%$ in dimensions and perimeter and in 30\% in TA area. Interestingly, in long-lasting severe FTR, the annulus becomes larger and more planar, losing its sphincteric action.

It is worth clarifying the difference between the mitral annulus (MA) and the TA. The anterior segment of the MA is anchored symmetrically to the two fibrous trigones and the posterior segment is made-up of a fibrous (though incomplete) support. Conversely, the septal TA is firmly anchored just at one point: the membranous septum with right fibrous trigone (central fibrous body) whereas the remaining antero-lateral-posterior contour consists of the hinge line of the leaflets attached to muscular and adipose tissue. Thus, while in the mitral annulus, the sphincteric contraction is more regular and the saddle-shaped configuration becomes more accentuated in systole, the sphincteric contraction of the tricuspid valve is much more complex, assuming a kind of "wave" motion depending on different degrees of contraction of the four segments and rotation and translation of the atrio-ventricular junction.

The TA excursion towards the apex is due to the longitudinal shortening of RV. Since, the part of the pericardial sac containing the apex of the heart is firmly anchored through dense connective tissue bands to the diaphragm, the systolic longitudinal contraction of right ventricle causes a descent of the atrio-ventricular plane. 3DTEE and CMR may clearly show annular dynamics (Fig 3.5). In the normal individual, the tricuspid annular plane systolic excursion (TAPSE), measured by 2D TTE in four-chamber view must be greater than $16 \mathrm{~mm}$.

\section{The right coronary artery}

Technically the right coronary artery (RCA) is not part of the TA. But, because of its proximity to the annulus and the potential complications that can occur during surgical and percutaneous transcatheter 
tricuspid valve repair, we include a brief description of the RCA course and its anatomical relationship with TA as a useful adjunct to this chapter.

With the exception of the septal portion, right coronary artery (RCA) runs within the atrioventricular groove encircling the entire parietal attachment of the tricuspid leaflets. The course of RCA within the atrio-ventricular groove is undulating and the distance between the RCA and the TA is variable. Several pathological and CT studies confirm the tendency of RCA to run relatively further away from TA in its anterior aspect (up to $20 \mathrm{~mm}$ ) and closer to the TA in its posterior aspect (less than $5 \mathrm{~mm}$ ).

The precise position in each segment between the RCA and the hinge line of leaflets, is a crucial information for both cardiac surgeons and interventional cardiologists. Direct injury by acute entrapment of RCA due to surgical sutures applied for ring fixation or RCA damage caused by annulus plication are rare but potentially life-threatening complications presenting as cardiogenic shock (due to acute right ventricle failure) or electrical instability. These complications are likely to occur especially in the case of DeVega annuloplasty or if the expected anatomical distances between RCA and TA are greatly altered by a very large TA. Impingement of the artery by pledgets or anchoring system during TV transcatheter annuloplasty is also a rare but potentially serious complication. There is no doubt that CT is the best imaging modality to visualize the particular anatomical relationship between RCA and TA (Fig. 3.6 A, B). In some cases the anterior aspect of RCA can be seen using 2D TEE trans-gastric short axis view (Fig. $3.6 \mathrm{C}$ ). 


\section{Leaflets}

In the classic anatomical books and journals the TV has been traditionally described as having three leaflets (anterior, posterior and septal) separated by three main indentations. The anterior leaflet is the largest, the most mobile and has a semicircular shape. The hinge line of the leaflet extends from the antero-septal commissure to the antero-posterior commissure. Given its size the anterior leaflet contributes the most to the competency of the valve. The posterior leaflet is smaller than the anterior, has a trapezoidal shape and it is attached to the posterior segment of the annulus. Finally, the septal leaflet is the smallest, roughly rectangular shaped and it is attached to the septal segment of the annulus. Anatomically, apart from being non-linear, the antero-superior part of its hinge line crosses the thinnest part of the cardiac septum which comprises of fibrous tissue and is termed the membranous septum (Fig. 3.4 C,D). Owing to the more apically located TV hinge line relative to that of the MV, one portion of the membranous septum separates the right atrium from the left ventricular outflow tract while the adjoining portion separates the two ventricles. Thus, the membranous septum has an atrioventricular and an interventricular component (see Chapter 4). The atrioventricular part adjoins the right fibrous trigone of the aorto-mitral curtain, forming the central fibrous body. This is an important landmark for the location of the atrioventricular conduction bundle of His. Furthermore, the part of the septal leaflet at the membranous septum may take various morphologies e.g. a small scallop, a bubble, or is partially or totally deficient thereby potentially leaving a gap in the closure line.

The leaflets are separated by deep incisures called commissures at three locations: antero-septal, antero-posterior, and posterior-septal. These commissures divide the valvular tissue into three main leaflets. Similar to the mitral valve, the three commissures do not reach to the hinge line. Thus, from a strictly anatomical point of view, the part of the leaflets toward the hingeline is a continuous veil of valvar tissue. The commissural tissue may be a simple strip or may assume the form of a small commissural scallop. Characteristic fanlike chordae are attached to their free edges. Each leaflet is has a basal zone of few millimeters near the hinge line, a clear zone where the leaflet is thin and translucent, and toward the free edge is a rough zone where chordae tendineae are attached on the underside. The rough zone corresponds on its atrial side to the coaptation area. The relatively large coaptation surface assures that in systole there is nearly perfect competence of the valve. 
As previously mentioned, the tricuspid valve morphology is hugely variable among individuals. This variability is particularly accentuated for the leaflets. Indeed, the entire tricuspid veil may be divided by indentations, which often do not conform to the classical scheme of three leaflets. Some authors describe TV as having only two leaflets: septal and mural, an anatomical architecture that mirrors the mitral valve, where the antero-posterior commissure is diminished or lacking. Whereas the septal leaflet is hinged to the most robust and immobile part of the TA, the mural leaflet hangs down from the TA that is related to the relatively mobile free wall. This part of the TA is characterized by significant changes in size and shape during the cardiac cycle; therefore, the mural leaflet is required to have indentations to adapt the leaflet to the changeable annular area and to ensure opening of leaflet which otherwise would be restricted because of long inter-commissural length. Indeed, quite often there is more than one indentation dividing the mural leaflet into up to six segments or scallops (Fig. 3.7). 


\section{Imaging techniques}

Both CT and CMR have several limitations in visualizing tricuspid leaflets: CT must use a dedicated acquisition protocol to optimally opacify the volume around the tricuspid leaflets. Furthermore, visualization of leaflets' motion requires ECG-gated retrospective acquisition with a significant increase of radiation exposure. Finally, the frame rate (i.e. number of images per second) is not optimal and inferior to the other imaging techniques (up to 15 frames per second with the latest CT machines). However, when the space around the leaflets and in the right ventricle is opacified enough, CT, because of its high spatial resolution, can visualize with an unbeatable clarity fine anatomical details of right ventricular structures such as chordae tendinea and papillary muscles (see below). Being thin and fast moving, images of tricuspid leaflets provided by CMR are often affected by blurring and ghost artifacts which make the leaflets almost indistinct (Fig.3.8).

Echocardiography is undoubtedly the first line imaging technique to visualize the TV leaflets in realtime. Both transthoracic and transesophageal echocardiography are able to visualize the valve from several different views. It must be emphasized that 2D TTE and TEE use tomographic cuts that perpendicularly transect the leaflets, thus their images represent only thin slices of leaflet tissue and only very few sections are able to transect the three leaflets simultaneously. In general, in normal individuals where the TA is elliptical echocardiographic images of TV are of medium quality, while in patients with large TA the images of the valve are unquestionably better.

Mid-esophageal 2D TEE should provide cross-sectional images of leaflets of higher quality than the equivalent cross sections of 2D TTE as is usual for the mitral valve (MV). However, MV leaflets are relatively thick, very close to the esophagus and their position in systole is almost perpendicular to the ultrasound beam. This fortunate combination of factors produces strong specular echoes that allow imaging from mid-esophagus to produce, on average, images of the MV of a quality superior to the corresponding transthoracic views. Conversely, the leaflets of TV are thinner than those of MV, further away from the mid-esophagus, and oblique with respect to the direction of ultrasound beam. This unfortunate combination of factors often produces TEE images of tricuspid leaflets as a line with several artifactual interruptions (drop-out artifacts) most likely because the majority of echoes returning from tricuspid leaflets are scattered and therefore not strong enough to significantly contribute to the reconstruction of the final 2D image. However, transgastric TEE views provide TV images which on 
average are of a quality superior to that of mid-esophageal, simply because the tip of the transducer is closer to the TV.

Three-dimensional echocardiography has the potential to image the entire surface of the tricuspid leaflets in an "en face" perspective mimicking the anatomic specimens. In patients with an optimal echocardiographic window, 3D TTE may provide diagnostic quality images from the apical four-chamber view. However, because of blurring or amplification artifacts, 3D TTE usually shows leaflets thicker than they really are. This phenomenon is less pronounced with 3D TEE. However, as for 2D TEE, 3D TEE suffers from drop-out artifacts due to the thinness of the leaflets and their obliquity with respect to the ultrasound beam. 3D TEE dropout artifacts appear as small holes on the surface of leaflets. The figure 3.9 is a collage of tricuspid images obtained with 2D TTE and TEE and with 3D TEE.

The septal leaflet deserves special attention. It is attached posteriorly to the muscular and anteriorly to membranous septum. Typically, the hinge line of the septal leaflet is apically displaced relative to the hinge line of the anterior mitral leaflet and this feature is always associated with the tricuspid valve, meaning that it can be used to recognize a morphologically right ventricle even when the chamber is on the wrong side of the ventricular mass. The mean difference in levels (offset) between the two hinge lines should not exceed 8-9 $\mathrm{mm}$ in the normal heart. An exaggerated offset could suggest Ebstein malformation. Contrary to the other leaflets, this leaflet has multiple chordae tendineae attaching it directly to the interventricular septum rendering it the least mobile of the leaflets. Notably, this septal attachment is probably the most constant feature of the tricuspid valve. Either TEE or CT scan can beautifully illustrate this anatomical peculiarity (Fig. 3.10). Furthermore, the septal leaflet extends antero-superiorly away from the membranous septum and the muscular ventricular septum because the commissure supporting the antero-septal commissure is attached to a small papillary muscle that arises from the posterior limb of the trabecular septomarginalis (TSM). This short part of the TA, no longer septal, relates instead to the aortic segment of the surgical view (Fig. 3.3). 


\section{The atrio-ventricular junction}

The septal leaflet is an integral part of the atrio-ventricular junction. This is a characteristic area where the interatrial and interventricular septa join with the anterior leaflet of mitral valve and the septal leaflet of tricuspid valve. This region will be discussed extensively in chapter 4

\section{The subvalvular apparatus}

The suspension apparatus comprises the chordae tendineae and papillary muscles (PM). As for other tricuspid valve structures, PMs in the right ventricle are highly variable in number, position and insertion of chordae, unlike the mitral valve. Classically, papillary muscles are described as being organized in three groups: anterior, posterior and septal. The anterior PM is the largest and the most constant being found in practically $100 \%$ of cases. It arises from the moderator band (MB) or the anterior wall at a level approximately one-third of ventricular length from the apex. There may be one papillary muscle with two or three heads or a group of smaller papillary muscles clustered together (Fig. 3.11). The chordae attach on the free margin and rough zones of the anterior and posterior leaflet and on the anteroposterior commissure. Particularly interesting is its anatomical relationship with the MB. Indeed, the MB originates from the body of the trabecula septo-marginalis (TSM), and, crosses the right ventricular cavity to insert into the RV anterior wall from where the anterior papillary muscle takes origin. The "triad" of TSM, MB and anterior PM, forms a "U-shaped" structure that demarcates the inflow from the outflow zones of the right ventricle. The posterior PM arising from the posterior wall is least consistent, usually seen as separate slender papillary muscles with chordae inserting to the free margin and rough zone. Short chordae attaching the basal part of the leaflet to the ventricular wall are seen in some hearts. As described previously, the antero-septal commissure is supported by a commissural chord attached to the medial (or conal/septal) papillary muscle also known as the muscle of Lancisi. This PM is small, singlular or multiple, implanted on the TSM. The spatial distribution of chordae tendineae mimics the mitral counterpart with marginal, secondary and basal chordae. As mentioned above, the TV characteristically has chordae tendineae directly arising from the interventricular septum to anchor the septal leaflet. 


\section{Pathoanatomy of Functional Tricuspid Regurgitation}

\section{Functional tricuspid regurgitation (FTR)}

FTR has been neglected for long time on the basis and assumption that it would simply disappear (or become irrelevant) at the time of mitral valve (MV) surgery. This long-held belief, derived from a study of Braunwald and Roger published in 1967, has promoted in the past a conservative surgical attitude (do not touch the valve). Nowadays, there are clear evidences that, despite successful left-heart surgery with valve repair/replacement, more than one half of patients with untreated FTR develops progressive valve dysfunction. Moreover, surgical series have demonstrated that any early reduction in TR following mitral surgery may be temporary, and may evolve over time to more severe FTR. On the other hand, patients with severe FTR after left-heart surgery, remain, despite the underlying TV disease, mildly symptomatic, or even asymptomatic for long periods, until overt right ventricular dysfunction occurs. As a consequence, repeat surgery for TV repair is often reserved for patients who are older, with several comorbidities, and, frequently, at the end-stage of right heart failure. Not surprisingly, surgical mortality for an isolated reintervention of FTR repair/replacement performed years after left-heart surgery is higher than for any other single valve repair/replacement. The European guidelines recommend tricuspid repair during left-heart surgery in the presence of severe FTR (class I; level of evidence C), moderate primary (organic) TR (II a; C), and $\geq$ mild TR with annular dilatation ( $\geq 40 \mathrm{~mm}$ ) (II a; C). The level " $\mathrm{C}$ " of evidence means that there is not unanimous consensus among experts. The lack of strong evidences has probably contributed to the considerable undertreatment of FTR. Thus, it is still unclear which criteria should be applied for TV repair in patients undergoing MV surgery. Although no longer forgotten, TV remains an "enigmatic" valve. We can prudently conclude that FTR is an insidious, unpredictable and progressive disease leading to right heart failure and eventually to death if not treated at its early stages. Nowadays, a "prophylactic" repair of TV during left side surgery is an accepted surgical option even for patients with moderate regurgitation when annular dilation is $>40$ $\mathrm{mm}$. The German Transcatheter Mitral Valve Interventions (TRAMI) registry analyzed the prognostic impact that the untreated FTR may have in patients undergoing percutaneous transcatheter procedures. The study found patients with severe TR had elevated rates of atrial fibrillation, pulmonary hypertension, residual mitral regurgitation and decreased survival rates compared to patients without 
severe FMR. The negative impact that untreated FTR may have on these patients and the high mortality of a surgical option, have highlighted the need to develop solutions for transcatheter tricuspid valve repair. Consequently, several catheter devices are currently under careful evaluation. Although there is still a lack of clinical data to support the efficacy of these transcatheter procedures, transcatheter devices in selected non-surgical patients may ultimately alleviate symptoms.

FTR represents the most frequent type of TV regurgitation, accounting for $90 \%$ of all severe TV regurgitation whereas other causes of TV regurgitation are far less common. Knowledge of normal TV anatomy underpins a deeper understanding of the pathoanatomy of TV leading to FTR. As with functional mitral regurgitation, several mechanisms play a role in determining FTR including annular dilation, right atrial and right ventricle dilation, papillary muscles displacement and leaflets tethering and tenting (Fig. 3.12).

The peculiar anatomy of TA results in an "asymmetric" dilation occurring mainly on the "free wall" hinge line, where the anterior and posterior leaflets are suspended. It is therefore not surprising that, in some patients with long-standing atrial fibrillation (AF), atrial remodeling may produce a direct effect on the annular hinge line leading to FTR. As the annulus dilates, posterior and anterior leaflets are pulled away from the septal leaflet and from each other thereby diminishing the apposition zone between leaflets. In animal models, only a $40 \%$ annular dilation may cause a regurgitant orifice with significant FTR. The susceptibility of the TV to annular dilation and its prevalence compared with mitral annulus in patients with $A F$ is explained by the lack of accretions of strong fibrous tissue supporting the TA. The observation that some patients with long standing AF do not have FTR is not yet well clarified. Individual tendency in developing right atrial remodeling or variations in leaflet size may explain these contradictory findings. Being the largest and the most mobile, the anterior leaflet may compensate for the annular dilatation, at least in the early stages. Patients with long-standing AF and FTR may not have enough leaflet tissue to adequately cover the increased orifice size. In case of severe FTR, surgical strategy includes either downsizing ring annuloplasty or leaflet augmentation.

More advanced FTR evokes a more complex interplay of factors such as annular, atrial, and ventricular enlargement. The RV dilation determines displacement of PMs mainly towards anterior-lateral and apical directions. The chordae arising from anterior PM, tether the anterior leaflet, the adjacent segments of the anterior and septal leaflets and the antero-posterior commissure, while chordae arising from the posterior PM tether the posterior leaflet and posterior-septal commissure. PM 
displacement leads to a loss of leaflets coaptation, which in turn worsens the FTR with further RV enlargement resulting in a deleterious vicious circle. As in functional mitral regurgitation, leaflet tenting is the effect of tethering and a sign of severe TR. In this scenario other pathological changes occur: the TA loses the oval aspect and the saddle-shaped configuration becoming round and flat and the sphincteric action diminishes. These pathological changes increase the distance between the hinge line and PMs, exacerbating the tethering on the leaflets. An effort to reorganize the complex mechanisms of FTR has been made by Dreyfus et al. The authors reclassified FTR in three stages roughly corresponding to the evolution of the disease: stage 1, mild FTR, annular diameter $<40 \mathrm{~mm}$, normal leaflet apposition; stage 2: moderate FTR, leaflets coaptation limited at the edges; stages 3: severe FTR annular diameter $>40 \mathrm{~mm}$, absent coaptation. The authors recommend medical therapy for stage 1 , tricuspid annuloplasty for stage 2 and tricuspid annuloplasty plus leaflet augmentation for stage 3 . 


\section{Figure legend}

Fig.3.1 (A) Anatomical specimen cut in four-chamber plane. The figure shows as the epicardial fibroadipose tissue (EAT) deeply penetrates (dotted line) in the atrioventricular groove up to the leaflet hinge line (arrow) (B). The corresponding histological section with myocardium stained red and fibrous tissue in EAT in green. Adipose tissue appears as voids in the groove. The arrow points at the hinge line of tricuspid leaflet. (C) 2D echocardiographic four chamber view. The area in the red box is magnified in panel B. (C) CT four chamber view. The area in the red box is magnified in panel D. Both imaging techniques are not able to distinguish EAT from surrounding structures. $\mathrm{RA}=$ right atrium; RV = right ventricle; RCA = right coronary artery.

Fig 3.2 Cardiac magnetic resonance cine-sequence still frame obtained with steady-state free procession (SSFP) in four chamber view (A, B) and in right ventricular inflow (C, D) view. The areas in the red box in the panel $A$ and $C$ are magnified in panel $B$ and $D$. (B) The image clearly shows the juxtaposition of the four components that form the tricuspid annulus: right atrial wall (RAW), right ventricular wall (RVW), epicardial fibro-adipose tissue (EAT) and the hinge line of tricuspid leaflet. (D) The image shows how deeply the EAT penetrates into the atrioventricular groove. E and F are corresponding cuts through anatomic specimens to show the hinge of the tricuspid valve leaflet (white arrow) and the deep excursion of EAT into the right atrioventricular groove. RA = right atrium, RV = right ventricle; $L A=$ left atrium; $L V=$ left ventricle, $R A A=$ right atrial appendage.

Fig. 3.3 (A) Anatomic specimen showing the base of the heart from the atrial perspective. The area in the red box is magnified in panel $B$ to show the surgical segmentation of the tricuspid annulus. (C) 3D TEE image obtained from trans-gastric view and oriented similar to the anatomical specimen in panel A. The area in the red box is magnified in panel $D$ showing the surgical segmentation. The double headed red arrow points at the distance between the anterior segment of the TA and the aortic root. Ao = Aorta; $\mathrm{PV}=$ pulmonary valve; $\mathrm{MV}=$ mitral valve; $\mathrm{TV}=$ tricuspid valve; $\mathrm{S}=$ septal leaflet; $\mathrm{A}=$ anterior leaflet; $\mathrm{P}=$ posterior leaflet. 
Fig. 3.4 (A) anatomic specimen and (B) 3D TEE in similar orientation with the position of the atrioventricular node (AVN), the membranous septum (MS) and His bundle superimposed. The yellow dotted curve line marks the line for placement of surgical sutures. The tricuspid rings are manufactured without the septal segment to avoid injuring the atrioventricular conduction system.

(C) View of the septal surface of a heart specimen with the TV opened to show the non-linear hinge line (blue broken line) of the septal leaflet (S) in situ with its most apical attachment (blue arrow) and highest attachment (open arrow). The antero-superior (AS) and poster-septal (PS) commissures (comm) are indicated. Note the additional scallop $\left(^{*}\right)$ in the posterior leaflet (P). (D) this enlargement highlights the membranous septum (red broken line) crossed by the hinge line. Leaflet tissue partially covers the interventricular component of the membranous septum and there is a gap (triangle), not uncommonly seen in normal hearts. The antero-septal commissure is supported by a diminutive medial papillary muscle in this heart (white arrow). Thus, while a large portion of the septal leaflet has attachments to the septum (small arrows), a small portion is away from the septum. $A=$ anterior leaflet, APM= anterior papillary muscle, AVN= AV node and His, CS- coronary sinus.

Fig. 3.5. 2D TOE transgastric perspective in (A) diastole and (B) systole, clearly showing the reduction in size of TA. (C, D) CMR balanced steady-state free procession (SSFP) sequences four-chamber cut showing the displacement of the TA towards the apex. The dotted red line represents the plane of TA and the double-head arrow the distance between the plane and the right ventricle apex in (C) diastole and in (D) systole. RA= Right atrium; RV = Right ventricle

Fig. 3.6 (A) volume rendering CT images showing the tortuous course of right coronary artery (RCA). (B) Maximum intensity projection showing the RCA encircling the annulus of the tricuspid valve (blue colored ellipse). (C) 2D TEE transgastric short axis view showing the anterior aspect of the course of RCA. The double-headed arrow points at the distance between the right ventricle (RV) and the RCA. RCS= right coronary aortic sinus

Fig. 3.7 (A-D) anatomic specimens of the tricuspid valve showing the wide variability of leaflet morphology. ${ }^{*}=$ scallops 
Figure 3.8. Images of tricuspid leaflets (red circles) provided by (A) computed tomography and (B) cardiac magnetic resonance (see text). $R A=$ right atrium; $L A=$ left atrium; $R V=$ right ventricle; $L V=$ left ventricle.

Fig. 3.9 a collage of tricuspid images obtained from (A-D) transthoracic 2D echocardiography; (C-D) 2D transesophageal echocardiography and (I-N) 3D transesophageal echocardiography. In cross sectional 2D echocardiography while the septal leaflet $(S)$ is always recognisable due to its constant relationship with the septum and the aorta, anterior $(A)$ and posterior $(P)$ leaflets cannot be detected with certainty using cross-sectional planes, given the extreme variability of the size and number. In such a case we prefer label the posterior and anterior leaflets as mural (M) leaflet. In the figure, scallops of mural leaflet are marked with asterisks.

Fig. 3.10 (A, B) CT scan, (C-D) 2D TEE and (E, F) anatomic specimen. The area in the white square of panels A, C, E is magnified in panels B, D, F respectively. Images show the septal leaflet anchored with short chordae tendineae directly on the interventricular septum (IVS).

Figure $3.11(A, B, C) C T$ multiplanar reconstruction images showing the right anterior papillary muscle (APM). (D, E, F) 3D TEE showing APM and posterior papillary muscles (PPM). (G. H. I) The anterior wall of the right ventricle has been removed from these 3 hearts to show some of the variations in the medial papillary muscle (blue arrows) and anterior papillary muscle (red arrows). $\mathrm{MB}=$ moderator band, $\mathrm{TSM}=$ trabecular septo-marginalis

Fig. 3.12 Two halves of a heart specimen with remarkably dilated right atrium(RA) and right ventricle(RV). The leaflet lengths from hinge line to free margin are too short to allow leaflet coaptation. The anterior papillary muscle (red arrow) is displaced and there is tethering of the anterior leaflet (black arrow). As mentioned, the TA is part of tricuspid valve but it is also a component of atrial and ventricular myocardium. If the right atrium or the right ventricle or both dilate, it is inevitable that the TA also dilates. 


\section{Suggested readings}

Nath J, Foster E, Heidenreich PA. Impact of tricuspid regurgitation on long-term survival. J. Am Coll Cardiol. 2004; 43:405-409

Braunwald NS, Ross J Jr, Morrow AG. Conservative management of tricuspid regurgitation in patients undergoing mitral valve replacement. Circulation. 1967 Apr; 35(4 Suppl):I63-9.

Kilic A, Saha-Chaudhuri P, Rankin JS, Conte JV. Trends and outcomes of tricuspid valve surgery in North America: an analysis of more than 50,000 patients from the Society of Thoracic Surgeons database. Ann Thorac Surg. 2013;96:1546-1552

Beckmann A, Funkat AK, Lewandowski J, Frie M, Schiller W,Hekmat K, Gummert JF, Mohr FW. Cardiac surgery in Germany during 2012: a report on behalf of the German Society for Thoracic and Cardiovascular Surgery. Thorac Cardiovasc Surg. 2014; 62:5-17.

Dreyfus GD, Martin RP, Chan JKM et al. Functional tricuspid regurgitation. A need to revise our understanding. J Am Coll Cardiol 2015;65:2331-6

Ohno Y, Attizzani GF, Capodanno D, Cannata S, Dipasqua F, Imme' S et al. Association of tricuspid regurgitation with clinical and echocardiographic outcomes after percutaneous mitral valve repair with the MitraClip System: 30-day and 12-month follow-up from the GRASP Registry. Eur Heart J Cardiovasc Imaging 2014;15:1246-55.

Virmani R. The tricuspid valve. Mayo Clin Proc. 1988; 63:943-946

Silver MD Lam JH. Ranganathan N. Wigle ED. Morphology of the human tricuspid valve, Circulation. $1971 ; 43: 333-348$ 
Dean JW, Ho SY, Rowland E, Mann J, Anderson RH.. Clinical anatomy of the atrioventricular junctions. J Am Coll Cardiol 1994; 24: 1725-1731.

Ueda A, McCarthy KP, Sanchez-Quintana D, Ho SY. Right atrial appendage and vestibule: further anatomic insights with implications for invasive electrophysiology. Europace 2013; 15:728-34.
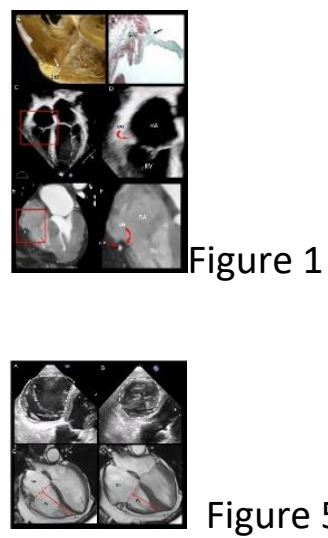

Figure 5
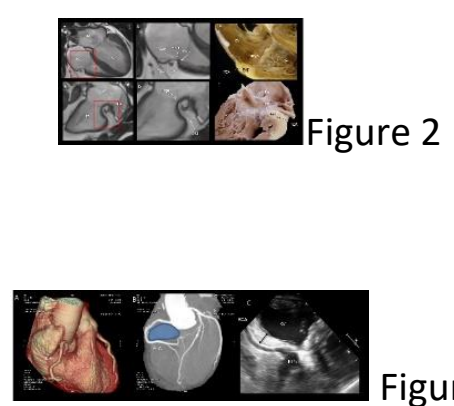

Figure 6

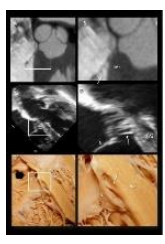

Figure 10

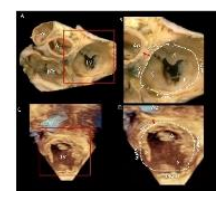

Figure 3

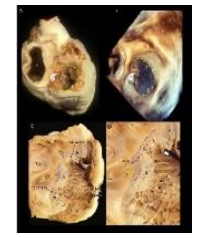

Figure 4
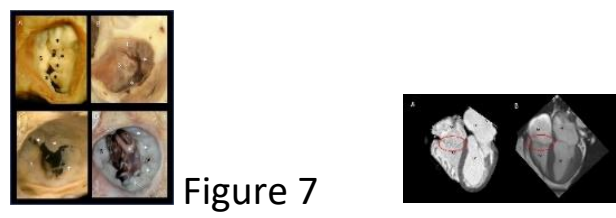

Figure 8

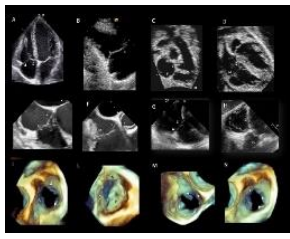

Figure 9
Figure 11

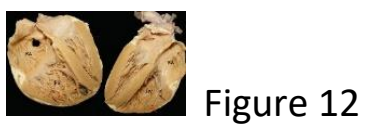

